ments. Then the spins and parities of these states have been deduced from angular distributions of the outgoing particles in the elastic scattering events. The new idea is to study pions (in this case negatively charged ones) scattered at $180^{\circ}$ from proton targets. When this backwards elastic differential cross-section is plotted against incident beam energy it exhibits a most striking structure, which can then be interpreted almost trivially in terms of resonance effects.

In the present experiment, where the incident energy range is from $1 \cdot 6$ to $5 \cdot 3 \mathrm{GeV} / c$, there is evidence for some nine peaks and dips (many of which stand out quite vividly) superimposed on a smooth and steadily decreasing background. The interpretation of the background effect is for the present purposes unimportant, although models for it in terms of elementary or alternatively Regge baryon exchange have accounted for it in a reasonable manner; all that one requires is that it be smooth and should not change sign. Then each pronounced peak or dip is interpreted as the effect of the pion and proton forming a resonant state during the scattering so as to produce constructive or destructive interference with the background term. As the incident energy at which a peak or dip occurs is trivially related to the mass of the resonances, these masses are effectively measured directly. Perhaps the most elegant feature, however, is that resonances of positive parity interfere constructively to produce peaks, and negative parity resonances produce dips, so that the parity of a resonance can be read off from the curve at a glance. The determination of such other parameters as elasticity or partial width is on much less firm foundation, but at least some indication of their values - to within factors of two, say-may be obtained by detailed analysis.

The present experiment has not only shown the value of the technique but has also provided the theoreticians with at least two big problems. First, there is a dramatic destructive interference (which the reader is urged to view for himself) at around $2 \cdot 2 \mathrm{GeV}$ resonance mass, in which the cross-section drops by two orders of magnitude. Second, there is a large narrow peak at $a$ resonance mass of about $3.25 \mathrm{GeV}$, suggesting a resonance there which has a width of the order of 1 per cent of its mass. That such a stable particle (half-life about $10^{-22}$ seconds) should exist at so high a mass will not be easy to explain.

\section{Italian Crystallographers}

\section{from a Correspondent}

THe newly founded Italian Crystallographic Association held its first general assembly in Perugia from January 11-13, 1968, in the Department of Chemistry of the University of Perugia, under the presidency of Professor F. Mazzi. The general assembly was combined with a symposium on the subject of four circle diffractometers. The two introductory lectures were given by B. T. M. Willis, who gave a general account of the whole subject.

Papers which followed covered the selection and operation of diffractometers. W. A. Wooster (Cambridge) maintained that the accuracy of $0.01^{\circ}$ usually quoted by makers of four circle diffractometers is not necessary, except on $\omega$ - and $2 \theta$-circles, when they are used for accurate measurement of lattice dimensions. He also discussed the relative merits of absolute-reading and difference-reading digitizers. J. Hornstra (Holland) described the PAILRED instrument, emphasizing the simplicity of a linear diffractometer which requires no computer or calculated angles. R. L. Beddoes (London) described the Hilger and Watts diffractometer and gave an account of its practical application. D. W. Beard (Cleveland, Ohio) dealt with crystal monochromators mounted in horizontal and vertical orientation and pointed out the relative merits of crystal reflected and filtered radiation. E. Curcio (Rome) described the GE diffractometer. A. J. H. Duisenberg (Holland) gave an account of the Nonius diffractometer and of some of the structural work carried out with it. E. R. Wölfel gave an account of two Stöe diffractometers. The first combines a Weissenberg photographic goniometer with a detector so that it becomes an equi-inclination diffractometer and the second is a four circle diffractometer with a special mounting for the X-ray tube. The last paper was given by H. Mayer (Germany), who described the Siemens four circle diffractometer, laying special stress on the accuracy of its mechanical construction.

\section{More Allosterism}

\section{from our Molecular Biology Correspondent}

IT has sometimes been difficult of late to avoid the disagreeable impression that the greater portion of biochemistry, if not indeed of chemistry, is gradually being engulfed by the allosteric hypothesis as particular cases of the universal truth. Among the all too large number of supposed allosteric phenomena in the current literature there is, however, an interesting group of articles on the enzyme aspartate transcarbamylase, which was studied by Gerhart and others, with the interesting upshot that the catalytic and regulatory sites occur on different sub-units.

This work has now been extended by Changeux, Gerhart and Schachman (Biochemistry, 17, 431 and 538; 1968), who have studied the binding of ligands to the protein and its sub-units. There are two catalytic and four regulatory sub-units, but it appears that four sites in all are present which will bind the substrate analogue, succinate, and four sites for the inhibitor, cytidine triphosphate (CTP). This situation can be accommodated by the capacious allosteric theory of regarding the enzyme as an isologous aggregate of four protomers (effective sub-units), each consisting of one regulatory sub-unit and half a catalytic subunit. In the intact protein, only the binding of succinate follows a sigmoidal saturation curve, so that the substrate sites evidently function in a co-operative manner. The reciprocal effect of substrate on inhibitor binding has also been demonstrated, uptake of CTP being partly reversed by succinate.

In the same issue of the journal, Gerhart and Schachman seek evidence for the conformational changes which are expected to accompany the attachment of ligands. It was in fact reported almost two years ago by Dratz and Calvin that the addition of succinate engenders in aspartate transcarbamylase a considerable change in optical rotatory dispersion which can reasonably be interpreted in terms of a difference in the content of $a$-helix. Gerhart and Schachman have, however, chosen a chemical approach-reactivity of sulphydryl groups. Reaction with mercurial leads to dissociation of the enzyme into its sub-units, but 